\title{
Simultaneously stimulating both brain hemispheres by rTMS in patients with unilateral brain lesions decreases interhemispheric asymmetry
}

\author{
Renhong He \\ Southern Medical University Nanfang Hospital \\ Yuhua Zhong \\ Southern Medical University Nanfang Hospital \\ Jimin Zhang \\ Southern Medical University nanfang hospital \\ Jianzhong Fan ( $\square$ fjznfyy168@163.com ) \\ Nanfang hospital, southern Medical University
}

\section{Research article}

Keywords: Brain symmetry index, Oscillation,Repetitive transcranial magnetic stimulation, Resting-state EEG, Unilateral brain lesions

Posted Date: November 12th, 2020

DOl: https://doi.org/10.21203/rs.3.rs-106005/v1

License: (1) This work is licensed under a Creative Commons Attribution 4.0 International License. Read Full License 


\section{Abstract}

Background: Interhemispheric asymmetry is common in brains with lesions and can be an adverse factor in the recovery of patients from neurological deficits. This study aimed to investigate the influence of repetitive transcranial magnetic stimulation (rTMS) on EEG power in patients with unilateral brain lesions by simultaneously stimulating both brain hemispheres and to elucidate asymmetrical changes in neurophysiological activity induced by rTMS.

Methods: Fourteen patients with unilateral brain lesions were treated with one session of $10 \mathrm{~Hz}$ rTMS over the vertex. Resting-state EEGs were recorded before and immediately after rTMS. The brain symmetry index (BSI), calculated from a fast Fourier transform, was used to quantify the power asymmetry in both hemispheres and paired channels over the whole frequency range and five frequency bands (delta, theta, alpha, beta and gamma bands).

Results: The results indicated that rTMS significantly reduced the BSI in patients with unilateral brain lesions over the entire frequency range $(t=2.767, P=0.016)$. Among the five frequency bands, $\mathrm{rTMS}$ only induced a noticeable decrease in the BSI in the delta band $(t=2.254, P=0.042)$. An analysis of different brain regions showed that significant changes in the BSI of the alpha band were found only in the posterior parietal lobe. EEG topographic mapping was employed to identify changes in cortical oscillations across brain regions, and demonstrated a decreased power of delta oscillations in the ipsilesional hemisphere and distinct cortical oscillations in the alpha band around the parietal-occipital lobe in the contralesional hemisphere.

Conclusions: When both brain hemispheres are activated simultaneously, rTMS decreases interhemispheric asymmetry primarily through reducing the delta wave in the lesioned hemisphere.

\section{Background}

Brain lesions caused by stroke and traumatic brain injury (TBI) can lead to neurological dysfunctions. A salient feature in brain-lesioned patients is a disruption in the interhemispheric excitability balance, which is partly responsible for abnormal cortical activity and neurological disorders. Ischemic stroke generates abnormal, slow-wave activity, particularly in the delta band, and attenuates normative, faster activity, particularly in the alpha and beta band. In addition, an increase in slow-wave activities in the unilateral lesioned-area negatively correlated with cognition [1]. Several lines of evidence have indicated that motor impairment correlated with an asymmetry in the alpha and beta bands where a loss of power in the alpha band and an increased power in the beta band in the ipsilesional central area signified a poor motor outcome [2, 3]. Similarly, the interhemispheric competition hypothesis suggested that balancing excitability between ipsilesional and contralesional hemispheres was an indicator of better behavior in stroke patients [4]. A treatment strategy highlights the importance of interhemispheric balancing, which shows that inhibition of cortical activity in the unaffected hemisphere has a therapeutic utility in stroke [5]. Therefore, modulating the abnormal cortical activity and rebalancing the interhemispheric activities is a meaningful approach to achieve neurological improvements in brain-lesioned patients.

Transcranial magnetic stimulation (TMS) is a non-invasive brain stimulation technique that can modulate cortical excitability and has shown promising applications in treating neurological disorders. Repetitive TMS 
(rTMS) is one type of TMS characterized by an output of many pulses, which can influence cortical activity temporally beyond the stimulation period and spatially beyond the stimulation site [6]. Low-frequency $(\leq 1 \mathrm{~Hz})$ and high-frequency ( $\geq 5 \mathrm{~Hz}$ ) rTMS protocols have a strong dichotomy in that the former can decrease cortical excitability and the latter usually produces a facilitating oscillatory activity [7]. Cortical oscillatory activities are generally assumed to play an important role in behavior, and oscillatory abnormalities may be one of the pathophysiological manifestations of neurological disorders [8]. For instance, high alpha oscillatory power is positively related to corticospinal excitability [9], and poor motor performance can result from decreased synchrony of alpha oscillations between the affected brain regions and the rest of the brain [10]. However, it remains unclear whether rTMS can modulate the interhemispherical asymmetry in different rhythm neuronal activities. Electroencephalograph (EEG) recordings show that the effects of rTMS on behavior are the consequence of oscillatory dynamics rather than creating a "virtual lesion" [11]. In comparison to a vegetative state, EEG responses are significantly different in a minimal consciousness state (MCS), which have higher cortical oscillations induced by rTMS [12]. These evidences indicate that EEG reactivity can be a validated method to help understand how rTMS affects brain activities.

In addition, ipsilesional and contralesional hemispheres have different roles in stroke recovery as increased excitability in the contralesional hemisphere can hamper the recovery by inhibiting the ipsilesional hemisphere [13]. A number of studies have investigated cortical activities after stimulation of the unilateral hemisphere by different rTMS protocols [14-16]. Ipsilesional activation, which is indicative of lesser bilateral activation, has significantly predicted larger treatment-induced behavioral gains for stroke patients [17]. However, there are no available studies that explore the changes of interhemispheric asymmetry in rhythmic neuronal activities in patients with unilateral brain lesions after both hemispheres were stimulated simultaneously by rTMS.

Therefore, we investigated the EEG after-effects in patients with unilateral brain lesions after both hemispheres were stimulated simultaneously by rTMS to determine whether rTMS could reduce the interhemispheric asymmetry and to define the contribution of the lesioned hemisphere or non-lesioned hemisphere in the reduction of interhemispheric asymmetry.

\section{Methods}

\section{Patients}

Fourteen patients were enrolled to participate in the study according to the inclusion and exclusion criteria. The inclusion criteria were: (1) age ranged between 18 years and 70 years; (2) diagnosis of a unilateral lesion proven by CT or MRI; (3) first-ever brain injury; (4) one-to-six months post-brain injury; and (5) no other neurological/psychiatric conditions. The exclusion criteria were: (1) a history of epilepsy within one month prior to recruitment; (2) a pacemaker or other metallic implants in head; (3) a skull defect; or (4) serious complications, such as heart failure or renal failure. The demographic and clinical features of all patients are described in Table 1. 
Table 1

Demographic and clinical features

\begin{tabular}{|llllll|}
\hline Patients & Age & Gender & Diagnosis & Affected side & Month since injury \\
\hline P1 & 28 & Male & Hemorrhagic & L & 1.5 \\
\hline P2 & 27 & Male & Hemorrhagic & L & 1 \\
\hline P3 & 70 & Male & Hemorrhagic & L & 3.5 \\
\hline P4 & 47 & Male & Hemorrhagic & L & 2 \\
\hline P5 & 43 & Male & Hemorrhagic & L & 1 \\
\hline P6 & 51 & Male & Hemorrhagic & L & 1.5 \\
\hline P7 & 29 & Male & Ischemia & L & 2 \\
\hline P8 & 37 & Male & Ischemia & L & 1 \\
\hline P9 & 52 & Male & Ischemia & L & 1 \\
\hline P10 & 56 & Male & Hemorrhagic & L & 6 \\
\hline P11 & 38 & Male & Hemorrhagic & L & 1 \\
\hline P12 & 64 & Male & Ischemia & L & 1 \\
\hline P13 & 46 & Male & Hemorrhagic & L & 1 \\
\hline P14 & 55 & Female & Hemorrhagic & L & 1 \\
\hline L: Left, P1-14: Patient 1-14 & & & \\
\hline
\end{tabular}

\section{Stimulation Procedures}

All patients received one session of $10 \mathrm{~Hz}$ rTMS at the $\mathrm{Cz}$ area (Cz position according to the International 1020 EEG System). TMS pulses were delivered using a stimulator (YRD, Wuhan, China) with a figure-of-eight focal coil. Surface electromyograph (sEMG) electrodes were taped along the belly-tendon of the bilateral abductor pollicis brevis. Before active rTMS, the stimulation intensity was determined based on the resting motor threshold (RMT), which was defined as the minimum output intensity that evoked a muscle contraction in at least 5 out of 10 stimulations with an amplitude of more than $50 \mu \mathrm{V}$ peak-to-peak in the relaxed abductor pollicis brevis of the affected side. When we tested the RMT, the patients wore the EEG cap to ensure an exact stimulation intensity. After the RMT was obtained, the coil was placed tangentially to the $\mathrm{Cz}$ scalp to produce electric current perpendicular to the central sulcus. It has previously been shown that both brain hemispheres can be activated simultaneously by one session of rTMS on $\mathrm{Cz}[18,19]$. One session of the rTMS procedure consisted of 1500 pulses $(10 \mathrm{~Hz}$, trains of 20 pulses over $2 \mathrm{~s}$ and at $30 \mathrm{~s}$ intervals) at an intensity of $100 \%$ of the RMT.

All patients in this study were required to attend three visits with at least 72 hours between visits. The first visit was designed to obtain the RMT and to set the individual's rTMS protocol. During the second and the last visit, 
the active session of rTMS and a sham-stimulation were randomly carried out. Pre-stimulation and poststimulation EEG signals were recorded in the active session. The sham-stimulation was carried out with the coil held at an angle of $90^{\circ}$, and pre-stimulation and post-stimulation EEG signals were record to confirm the effects of active rTMS.

\section{EEG data recording and preprocessing}

Resting-state EEGs were recorded to obtain at least $10 \mathrm{~min}$ of a stable signal before and immediately after the rTMS interventions in active and sham conditions. EEG data were acquired through the EEG System with a TMS-compatible amplifier (NuerOne, Bittium Bio-Signals Ltd, Finland) and an EEG Ag/AgCl cap with nineteen channels (GREENTEK, China). According to the International 10-20 EEG System, the nineteen electrodes were consisted of Fp1, Fp2, F7, F8, F3, F4, C3, C4, P3, P4, T3, T4, T5, T6, 01, 02, Fz, Cz and Pz. The FCz electrode was set as an online reference. The impedance of all electrodes was maintained below $5 \mathrm{k} \Omega$ and the sampling rate was $1000 \mathrm{~Hz}$. Patients laid in bed with their eyes closed while awake in a quiet room, and wore earplugs to reduce the noise disturbance induced by rTMS. During the rTMS session, the EEG cap was kept on the head and recorded the signals immediately after the session ended.

EEG data were processed offline using EEGLAB14.0 running in MATLAB2018. Data were referenced to an averaged reference and band-pass filtered between 0.5 and $48 \mathrm{~Hz}$. Drifting and non-obvious EEG signals were rejected based on a visual inspection and then the independent component analysis was carried out to remove artifact components, including eye blinks and muscle contractions.

\section{EEG data processing}

A fast Fourier transform ( $2 \mathrm{~s}$ Hamming) was applied to estimate the EEG power at different frequency bands. The absolute power was obtained for the delta $(0.5-4 \mathrm{~Hz})$, theta $(4-8 \mathrm{~Hz})$, alpha $(8-13 \mathrm{~Hz})$, beta $(13-30 \mathrm{~Hz})$, gamma $(30-40 \mathrm{~Hz})$ and whole $(0.5-40 \mathrm{~Hz})$ frequency bands of both hemispheres and all channels [20]. The brain symmetry index (BSI) of the five frequency bands was calculated for two hemispheres and paired channels. The equation for hemispheres was [BSI = Power (L_Hemi-R_Hemi)/Power (L_Hemi + R_Hemi)]. The equation for paired channels was $[\mathrm{BSI}=$ Power $(\mathrm{C} 3-\mathrm{C} 4) /$ Power $(\mathrm{C} 3+\mathrm{C} 4)]$. BSI $=0$ represents perfect symmetry and that a decrease in BSI corresponds to an increase in symmetry.

\section{Statistical Analyses}

A paired $t$-test was performed to compare the BSI of pre-rTMS and post-rTMS under the normal distribution at different frequency bands. Within-subject ANOVAs for repeated measure were applied to the comparisons among different sessions. A post-hoc Bonferroni's correction was used for multiple comparisons between sessions. The sphericity assumption was assessed with a Mauchly's test. Greenhouse-Geisser epsilon adjustments for non-sphericity were made where appropriate. The topographical mapping was plotted to show the cortical oscillatory power at different frequency bands. All data analyses and graphic designs were made in SPSS version 20, OriginPro 9, and MATLAB 2018.

\section{Results}


To determine if patients with unilateral brain lesions had interhemispheric asymmetry in different frequency bands, the BSI prior to the sham-control stimulation or the rTMS was examined in different frequency bands. We observed obvious interhemispheric asymmetry in different frequency bands. Further comparison among the active session, and before and after the sham-stimulation was done to determine whether confounding factors (e.g. noise and time) affected the BSI. No differences in the BSI were observed between hemispheres or between paired channels in all frequency bands after the sham-stimulation. In addition, no statistical differences were observed between the BSI in the sham-stimulation and the active session before rTMS intervention (Fig. 1).

\section{Effects of rTMS on interhemispheric asymmetry}

To elucidate the effects of rTMS on interhemispheric asymmetry, a paired $t$-test was performed to evaluate changes in the BSI. The results showed that the BSI was significantly decreased after one session of $\mathrm{rTMS}$ ( $t=$ $2.767, P=0.016)$. As shown in Fig. 2 , a more in-depth analysis of the different frequency bands revealed that the BSI of the delta band had a significant reduction $(t=2.254, P=0.042)$ induced by rTMS; however, there were no other significant after-effects on BSI induced by rTMS in the other four frequency bands.

\section{Effects of rTMS on asymmetry in paired channels}

We compared eight paired-channels at different frequency bands and found that rTMS significantly induced EEG after-effects in channels P3 \& P4 and 01 \& 02. Moreover, the BSI was significantly increased in the alpha band (Fig. 3) in those four channels. However, no significant BSI after-effects were observed in any of the other frequency bands in all channels (for details, see the supplementary files).

\section{Cortical oscillatory power at different frequency bands}

Figure 4 shows the distribution of the cortical oscillatory power at different frequency bands before and immediately after rTMS. The delta wave in most of the patients was largely localized in the lesioned area and rTMS reduced its oscillatory power. In addition, rTMS induced visible alpha oscillations around the posterior parietal area (P3 \& P4) in the contralesional hemisphere.

\section{Discussion}

In the present study, we performed one session of a $10 \mathrm{~Hz}$ rTMS protocol on patients with unilateral brain lesions to simultaneously stimulate both brain hemispheres to investigate how rTMS affects the interhemispheric asymmetry in these patients by measuring neurophysiological activity. We found that rTMS ameliorated the interhemispheric asymmetry in patients with unilateral brain lesions and this amelioration may be related to a reduction of delta waves in the ipsilesional hemisphere. Moreover, the rTMS protocol used in this study induced cortical oscillations around the alpha-predominant area (posterior parietal lobe) in the contralesional hemisphere.

Rhythmic oscillations, mainly consisting of the delta, theta, alpha, beta, and gamma frequency bands, are generated from groups of neurons and play a crucial role in functional cortical activities. These rhythmic oscillation changes are helpful in our understanding of brain function [8]. Brain oscillations reflect rhythmic fluctuations of local field potentials between excitatory and inhibitory states of neural populations [21]. During 
wakefulness, delta activity is almost absent in physiological conditions, but will appear in patients with brain injury. Alpha-band activity is the dominant oscillation in the awake human brain. High alpha power may indicate internally oriented brain states, and low alpha power may indicate externally oriented brain states [22]. In the healthy brain, the distribution and power of different frequency bands in the left and right hemispheres are the same, which is known as interhemispheric balancing.

In the brains of patients with unilateral brain lesions, it has been established that there is decreased cortical excitability in the brain-lesioned hemisphere and increased excitability in the contralesional hemisphere. Previous studies have provided evidence that this imbalance is an impedance to stroke recovery, which is further supported by the outcome improvement as a result of inhibiting the hyper-excitability of the contralesional side by brain stimulation techniques, such as transcranial direct current stimulation (tDCS) and rTMS [23, 24]. In line with the previous evidence, this study also found an apparent asymmetry between the ipsilesional and contralesional hemispheres in the brains of patients with unilateral brain lesions. In addition, it is noteworthy this asymmetry was not present in all frequency bands. However, brain lesions caused by stroke or TBI can disrupt the interhemispheric balancing and subsequently cause some adverse outcomes. Furthermore, stroke patients have significantly more interhemispheric asymmetries compared to healthy controls. For example, the BSI at early stages after stroke correlates with Fugl-Meyer motor scores in the recovery stage [25].

In this study, we found that one session of rTMS can ameliorate the brain asymmetry present in patients with unilateral brain lesions and has potential benefits on the restoration of interhemispheric balance in these patients. These conclusions are in line with a study that found that rTMS can reduce the neurophysiological imbalance during depression and result in improvements in neuropsychological test scores [26]. The asymmetry or imbalance as a result of unilateral brain injury is not only because of local lesions, but also because of the interaction of the contralesional side via the corpus callosum [27, 28]. In addition, many dysfunctions after brain injury, such as motor function [27], unilateral neglect [29], and aphasia [30], are reported to be related to the interhemispheric imbalance. Neuromodulation has been shown to reestablish the balance by either increasing the excitability of the ipsilesional hemisphere or inhibiting the cortical activities in the contralesional hemisphere.

An increase in slow-wave bands (delta and theta) in the lesioned hemisphere is acknowledged as a remarkable sign in patients with brain injury and is considered as a potential cause to the interhemispheric asymmetry [31, 32]. Our results also showed that activities of the delta band are mainly localized in the lesioned hemisphere. Higher delta activity indicates a worse outcome after brain injury and is typical of disorders of consciousness $[33,34]$. Importantly, our study showed that the interhemispheric asymmetry in the delta band was significantly reduced by one session of $10 \mathrm{~Hz}$ rTMS. Because the effects of rTMS can be observed not only in areas local to the stimulation site, but also in remote, anatomically- and/ or functionally-connected sites [35], we consider that the reduction of delta activity in the perilesional area is related to the activation of neuronal oscillations and the improvement of neural connectivity induced by rTMS. The results are supported by the Kamp et al. study [36] that shows that high-frequency rTMS can reduce delta-band activity in patients with schizophrenia. Therefore, we infer that the significant power reduction in the delta band in the ipsilesional hemisphere may be mostly responsible for the decreased interhemispheric asymmetry induced by rTMS in brain-lesioned patients. 
Furthermore, high-frequency rTMS has been shown to induce high-frequency oscillations, which can reduce the activity of slow-wave bands[37].

The EEG after-effects induced by rTMS were analyzed in different brain regions and we found that the alpha asymmetry in channels P3 \& P4 and 01 \& 02 was significantly changed after rTMS intervention. The topographic distribution also demonstrated that the alpha oscillatory power in the parietal-occipital area had a visible change after rTMS intervention. These results indicate that $10 \mathrm{~Hz}$ rTMS has a potential effect on modulating the cortical activities around the alpha-predominant area in the contralesional side. The neurological effects of rTMS have been shown to depend on the relationship between the frequency of stimulation and endogenous oscillatory activity $[38,39]$. Given that our study did not have experimental rTMS protocols at a different frequency, whether activating the alpha predominant area is associated with the frequency of $10 \mathrm{~Hz}$ is unknown. An increasing amount of evidence suggests that the effects of rTMS on cortical activity are due to the entrainment of frequency oscillations close to the frequency of stimulation [40, 41]. One recent publication has shown that the higher the individual alpha frequency (IAF) and the lower the absolute distance of the IAF from the rTMS frequency resulted in greater improvements of symptoms of depression following a six-week course of rTMS [42]. We tend to agree that the frequency of rTMS in this study that is close to the endogenous cortical rhythm is crucial for the entrainment of oscillation only in the parietaloccipital area, which implies that endogenous oscillation rhythm in a functional brain network may have a possibility to be a reference in choosing the frequency for rTMS protocols.

Certain limitations of our study should be addressed when interpreting the results. One limitation of the study is a relatively small sample size. The use of a repeated-measures analysis within-subjects allowed for the satisfactory control of the confound variables. Future studies using more subjects may be warranted for confirming the results. In addition, no functional tests were used to verify a therapeutic effect that resulted from the asymmetry reduction induced by rTMS. However, we are currently evaluating the therapeutic effects of rTMS on motor function. Finally, the different etiologies of brain injury in the patients of our study potentially decreased the consistency of data, which resulted in broad standard deviations.

\section{Conclusion}

In conclusion, this study demonstrates that $10 \mathrm{~Hz}$ rTMS can decrease the interhemispheric asymmetry in patients with unilateral brain lesions and induce alpha cortical oscillations in the parietal-occipital lobe. The alpha cortical oscillations are more likely in the contralesional hemisphere. The reduction of the delta band from the lesioned hemisphere may be the main contributor to the benefits induced by rTMS.

\section{Abbreviations}

BSI: Brain symmetry index; EEG: Electroencephalograph; rTMS: Repetitive transcranial magnetic stimulation; RMT: Resting motor threshold; sEMG: Surface electromyograph; TBI: Traumatic brain injury; TMS: Transcranial magnetic stimulation; tDCS: Transcranial direct current stimulation

\section{Declarations}


This study was conducted in accordance with the Declaration of Helsinki and approved by the Medical Ethics Committee of Nanfang Hospital, Southern Medical University. All patients and their families were informed about the procedure of this study and gave written informed consent.

\section{Consent for publication}

Not applicable

\section{Availability of data and materials}

The full datasets used and/or analysed during the current study available from the corresponding author on reasonable request.

\section{Competing interests}

The authors declare that the research has no competing financial interest.

\section{Funding}

This study was supported by the Technology Foundation of Guangdong Province (grant number: 2017B020247001) and the National Natural Science Foundation of China (grant number: 81802250). The funding body had no role in the design of the study and collection, analysis, and interpretation of data and in writing the manuscript.

\section{Author Contributions}

$H R H$ collected the data and drafted the manuscript. $Y H Z$ and $Z M Z$ were involved in the recruitment of the patients. JZF developed guided this study and revised the manuscript. All authors have read and approved the manuscript

\section{Acknowledgements}

Not applicable

\section{References}

1. Schleiger E, Sheikh N, Rowland T, Wong A, Read S, Finnigan S. Frontal EEG delta/alpha ratio and screening for post-stroke cognitive deficits: The power of four electrodes. Int J Psychophysiol. 2014;94:19-24. doi:10.1016/j.ijpsycho.2014.06.012.

2. Stinear CM. Prediction of motor recovery after stroke: advances in biomarkers. Lancet Neurol. 2017;16:826-36. doi:10.1016/S1474-4422(17)30283-1.

3. Thibaut A, Simis M, Battistella LR, Fanciullacci C, Bertolucci F, Huerta-Gutierrez R, et al. Using Brain Oscillations and Corticospinal Excitability to Understand and Predict Post-Stroke Motor Function. Front Neurol. 2017;8 MAY:1-8. doi:10.3389/fneur.2017.00187.

4. Corti M, Patten C, Triggs W. Repetitive Transcranial Magnetic Stimulation of Motor Cortex after Stroke. Am J Phys Med Rehabil. 2012;91:254-70. doi:10.1097/PHM.0b013e318228bf0c. 
5. Sebastianelli L, Versace V, Martignago S, Brigo F, Trinka E, Saltuari L, et al. Low-frequency rTMS of the unaffected hemisphere in stroke patients: A systematic review. Acta Neurol Scand. 2017;136:585-605. doi:10.1111/ane.12773.

6. Siebner H, Rothwell J. Transcranial magnetic stimulation: new insights into representational cortical plasticity. Exp Brain Res. 2003;148:1-16. doi:10.1007/s00221-002-1234-2.

7. Reid V. Transcranial magnetic stimulation. Phys Med Rehabil Clin N Am. 2003;14:307-25. doi:10.1016/S1047-9651(02)00120-1.

8. Assenza G, Capone F, di Biase L, Ferreri F, Florio L, Guerra A, et al. Oscillatory Activities in Neurological Disorders of Elderly: Biomarkers to Target for Neuromodulation. Front Aging Neurosci. 2017;9 JUN:1-18. doi:10.3389/fnagi.2017.00189.

9. Thies M, Zrenner C, Ziemann U, Bergmann TO. Sensorimotor mu-alpha power is positively related to corticospinal excitability. Brain Stimul. 2018;11:1119-22. doi:10.1016/j.brs.2018.06.006.

10. Dubovik S, Ptak R, Aboulafia T, Magnin C, Gillabert N, Allet L, et al. EEG alpha band synchrony predicts cognitive and motor performance in patients with ischemic stroke. Behav Neurol. 2013;26:187-9.

11. Johnson JS, Hamidi M, Postle BR. Using EEG to Explore How rTMS Produces Its Effects on Behavior. Brain Topogr. 2010;22:281-93. doi:10.1007/s10548-009-0118-1.

12. Xia X, Liu Y, Bai Y, Liu Z, Yang Y, Guo Y, et al. Long-lasting repetitive transcranial magnetic stimulation modulates electroencephalography oscillation in patients with disorders of consciousness. Neuroreport. 2017;28:1022-9. doi:10.1097/WNR.0000000000000886.

13. Dodd KC, Nair VA, Prabhakaran V. Role of the Contralesional vs. Ipsilesional Hemisphere in Stroke Recovery. Front Hum Neurosci. 2017;11 September:1-9.

14. McAllister CJ, Ronnqvist KC, Stanford IM, Woodhall GL, Furlong PL, Hall SD. Oscillatory Beta Activity Mediates Neuroplastic Effects of Motor Cortex Stimulation in Humans. J Neurosci. 2013;33:7919-27. doi:10.1523/JNEUROSCI.5624-12.2013.

15. Lee J, Lee A, Kim H, Shin M, Yun SM, Jung Y, et al. Different Brain Connectivity between Responders and Nonresponders to Dual-Mode Noninvasive Brain Stimulation over Bilateral Primary Motor Cortices in Stroke Patients. Neural Plast. 2019;2019:3826495. doi:10.1155/2019/3826495.

16. Park C, Chang WH, Yoo W-K, Shin Y-I, Kim ST, Kim Y-H. Brain topological correlates of motor performance changes after repetitive transcranial magnetic stimulation. Brain Connect. 2014;4:265-72. doi:10.1089/brain.2013.0187.

17. Burke Quinlan E, Dodakian L, See J, McKenzie A, Le V, Wojnowicz M, et al. Neural function, injury, and stroke subtype predict treatment gains after stroke. Ann Neurol. 2015;77:132-45. doi:10.1002/ana.24309.

18. de Araújo AVL, Barbosa VRN, Galdino GS, Fregni F, Massetti T, Fontes SL, et al. Effects of high-frequency transcranial magnetic stimulation on functional performance in individuals with incomplete spinal cord injury: study protocol for a randomized controlled trial. Trials. 2017;18:522. doi:10.1186/s13063-017-22801.

19. Kumru H, Benito-Penalva J, Valls-Sole J, Murillo N, Tormos JM, Flores C, et al. Placebo-controlled study of rTMS combined with Lokomat ${ }^{\circledR}$ gait training for treatment in subjects with motor incomplete spinal cord injury. Exp Brain Res. 2016;234:3447-55. doi:10.1007/s00221-016-4739-9. 
20. Okamura H, Jing H, Takigawa M. EEG Modification Induced by Repetitive Transcranial Magnetic Stimulation. J Clin Neurophysiol. 2001;18:318-25. doi:10.1097/00004691-200107000-00003.

21. HansImayr S, Gross J, Klimesch W, Shapiro KL. The role of alpha oscillations in temporal attention. Brain Res Rev. 2011;67:331-43. doi:10.1016/j.brainresrev.2011.04.002.

22. Jensen O, Mazaheri A. Shaping Functional Architecture by Oscillatory Alpha Activity: Gating by Inhibition. Front Hum Neurosci. 2010;4. doi:10.3389/fnhum.2010.00186.

23. Hertenstein E, Waibel E, Frase L, Riemann D, Feige B, Nitsche MA, et al. Modulation of creativity by transcranial direct current stimulation. Brain Stimul. 2019; xxxx:1-9. doi:10.1016/j.brs.2019.06.004.

24. Blesneag A V, Slăvoacă DF, Popa L, Stan AD, Jemna N, Isai Moldovan F, et al. Low-frequency rTMS in patients with subacute ischemic stroke: clinical evaluation of short and long-term outcomes and neurophysiological assessment of cortical excitability. J Med Life. 2015;8:378-87. http://www.ncbi.nlm.nih.gov/pubmed/26351545\%0Ahttp://www.pubmedcentral.nih.gov/articlerender.fcgi? artid=PMC4556924.

25. Agius Anastasi A, Falzon O, Camilleri K, Vella M, Muscat R. Brain Symmetry Index in Healthy and Stroke Patients for Assessment and Prognosis. Stroke Res Treat. 2017;2017:1-9. doi:10.1155/2017/8276136.

26. Spampinato C, Aguglia E, Concerto C, Pennisi M, Lanza G, Bella R, et al. Transcranial magnetic stimulation in the assessment of motor cortex excitability and treatment of drug-resistant major depression. IEEE Trans Neural Syst Rehabil Eng. 2013;21:391-403. doi:10.1109/TNSRE.2013.2256432.

27. Murase N, Duque J, Mazzocchio R, Cohen LG. Influence of interhemispheric interactions on motor function in chronic stroke. Ann Neurol. 2004;55:400-9. doi:10.1002/ana.10848.

28. Takeuchi N, Oouchida Y, Izumi S-I. Motor Control and Neural Plasticity through Interhemispheric Interactions. Neural Plast. 2012;2012:1-13. doi:10.1155/2012/823285.

29. Fu W, Cao L, Zhang Y, Huo S, Du J, Zhu L, et al. Continuous theta-burst stimulation may improve visuospatial neglect via modulating the attention network: a randomized controlled study. Top Stroke Rehabil. 2017;24:236-41. doi:10.1080/10749357.2016.1253139.

30. Rutar Gorišek V, Zupanc Isoski V, Belič A, Manouilidou C, Koritnik B, Bon J, et al. Beyond aphasia: Altered EEG connectivity in Broca's patients during working memory task. Brain Lang. 2016;163:10-21. doi:10.1016/j.bandl.2016.08.003.

31. Lu XC, Williams AJ, Tortella FC. Quantitative electroencephalography spectral analysis and topographic mapping in a rat model of middle cerebral artery occlusion. Neuropathol Appl Neurobiol. 2001;27:481-95. http://www.ncbi.nlm.nih.gov/pubmed/11903931. Accessed 2 Aug 2019.

32. Fanciullacci C, Bertolucci F, Lamola G, Panarese A, Artoni F, Micera S, et al. Delta Power Is Higher and More Symmetrical in Ischemic Stroke Patients with Cortical Involvement. Front Hum Neurosci. 2017;11 July:110. doi:10.3389/fnhum.2017.00385.

33. Butz M, Gross J, Timmermann L, Moll M, Freund H-J, Witte OW, et al. Perilesional pathological oscillatory activity in the magnetoencephalogram of patients with cortical brain lesions. Neurosci Lett. 2004;355:936. doi:10.1016/j.neulet.2003.10.065.

34. Leon-Carrion J, Martin-Rodriguez JF, Damas-Lopez J, Barroso y Martin JM, Dominguez-Morales MR. Brain function in the minimally conscious state: A quantitative neurophysiological study. Clin Neurophysiol. 2008;119:1506-14. doi:10.1016/j.clinph.2008.03.030. 
35. Di Lazzaro V, Dileone M, Pilato F, Capone F, Musumeci G, Ranieri F, et al. Modulation of motor cortex neuronal networks by rTMS: comparison of local and remote effects of six different protocols of stimulation. J Neurophysiol. 2011;105:2150-6. doi:10.1152/jn.00781.2010.

36. Kamp D, Brinkmeyer J, Agelink MW, Habakuck M, Mobascher A, Wölwer W, et al. High frequency repetitive transcranial magnetic stimulation (rTMS) reduces EEG-hypofrontality in patients with schizophrenia. Psychiatry Res. 2016;236:199-201. doi:10.1016/j.psychres.2016.01.007.

37. Pisani LR, Naro A, Leo A, Aricò I, Pisani F, Silvestri R, et al. Repetitive transcranial magnetic stimulation induced slow wave activity modification: A possible role in disorder of consciousness differential diagnosis? Conscious Cogn. 2015;38:1-8. doi:10.1016/j.concog.2015.09.012.

38. Hamidi M. Repetitive transcranial magnetic stimulation affects behavior by biasing endogenous cortical oscillations. Front Integr Neurosci. 2009;3:1-2. doi:10.3389/neuro.07.014.2009.

39. Klimesch W, Sauseng P, Gerloff $C$. Enhancing cognitive performance with repetitive transcranial magnetic stimulation at human individual alpha frequency. Eur J Neurosci. 2003;17:1129-33.

http://www.ncbi.nlm.nih.gov/pubmed/12653991. Accessed 27 Jul 2019.

40. Thut G, Veniero D, Romei V, Miniussi C, Schyns P, Gross J. Rhythmic TMS causes local entrainment of natural oscillatory signatures. Curr Biol. 2011;21:1176-85. doi:10.1016/j.cub.2011.05.049.

41. Romei V, Bauer M, Brooks JL, Economides M, Penny W, Thut G, et al. Causal evidence that intrinsic betafrequency is relevant for enhanced signal propagation in the motor system as shown through rhythmic TMS. Neuroimage. 2016;126:120-30. doi:10.1016/j.neuroimage.2015.11.020.

42. Corlier J, Carpenter LL, Wilson AC, Tirrell E, Gobin AP, Kavanaugh B, et al. The relationship between individual alpha peak frequency and clinical outcome with repetitive Transcranial Magnetic Stimulation (rTMS) treatment of Major Depressive Disorder (MDD). Brain Stimul. 2019;12:543-4. doi:10.1016/j.brs.2019.07.018.

\section{Figures}




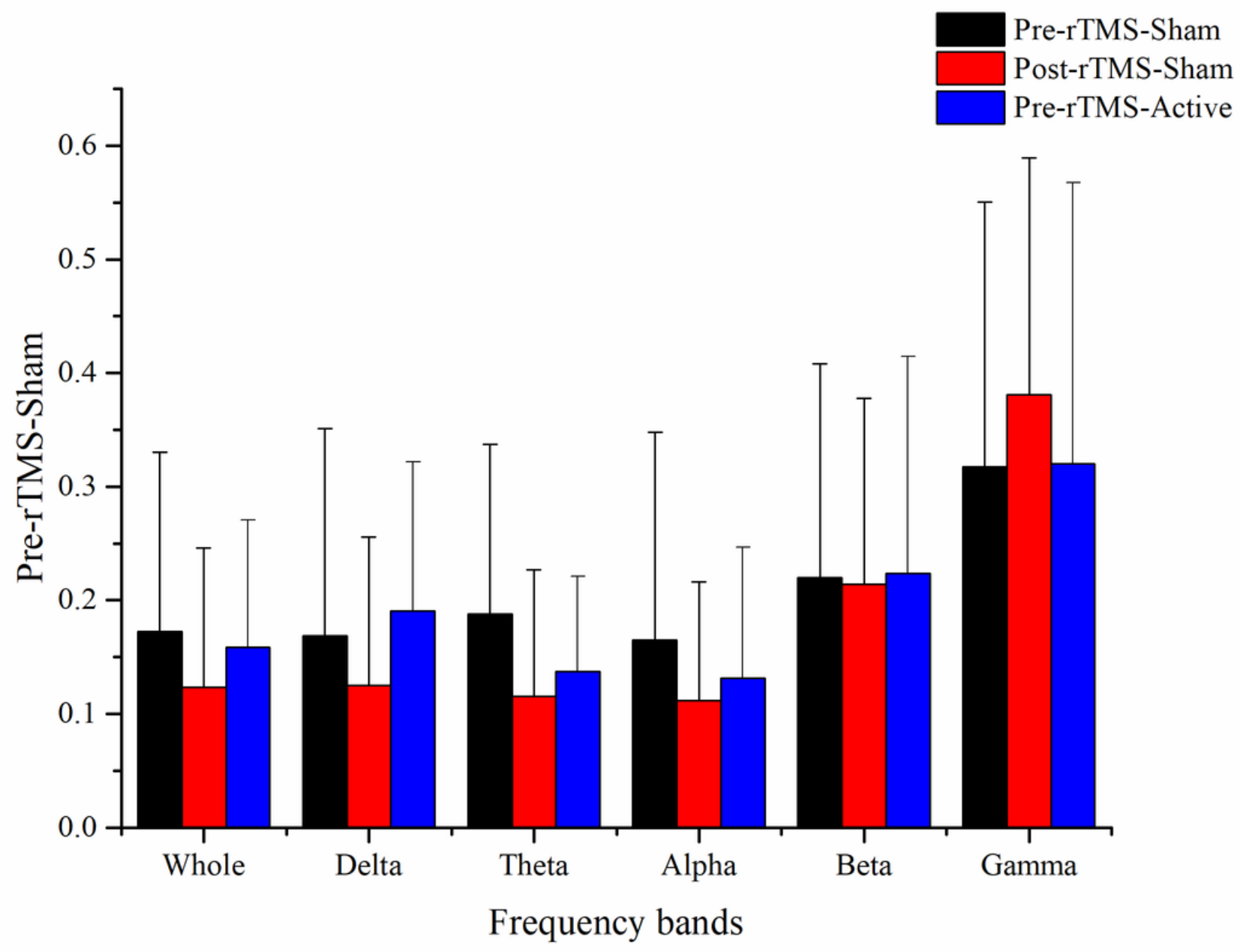

Figure 1

The comparison between sham and active sessions. No significant difference in BSI was found among the prerTMS and post-rTMS in the sham-stimulation sessions and the pre-rTMS in the active sessions. 


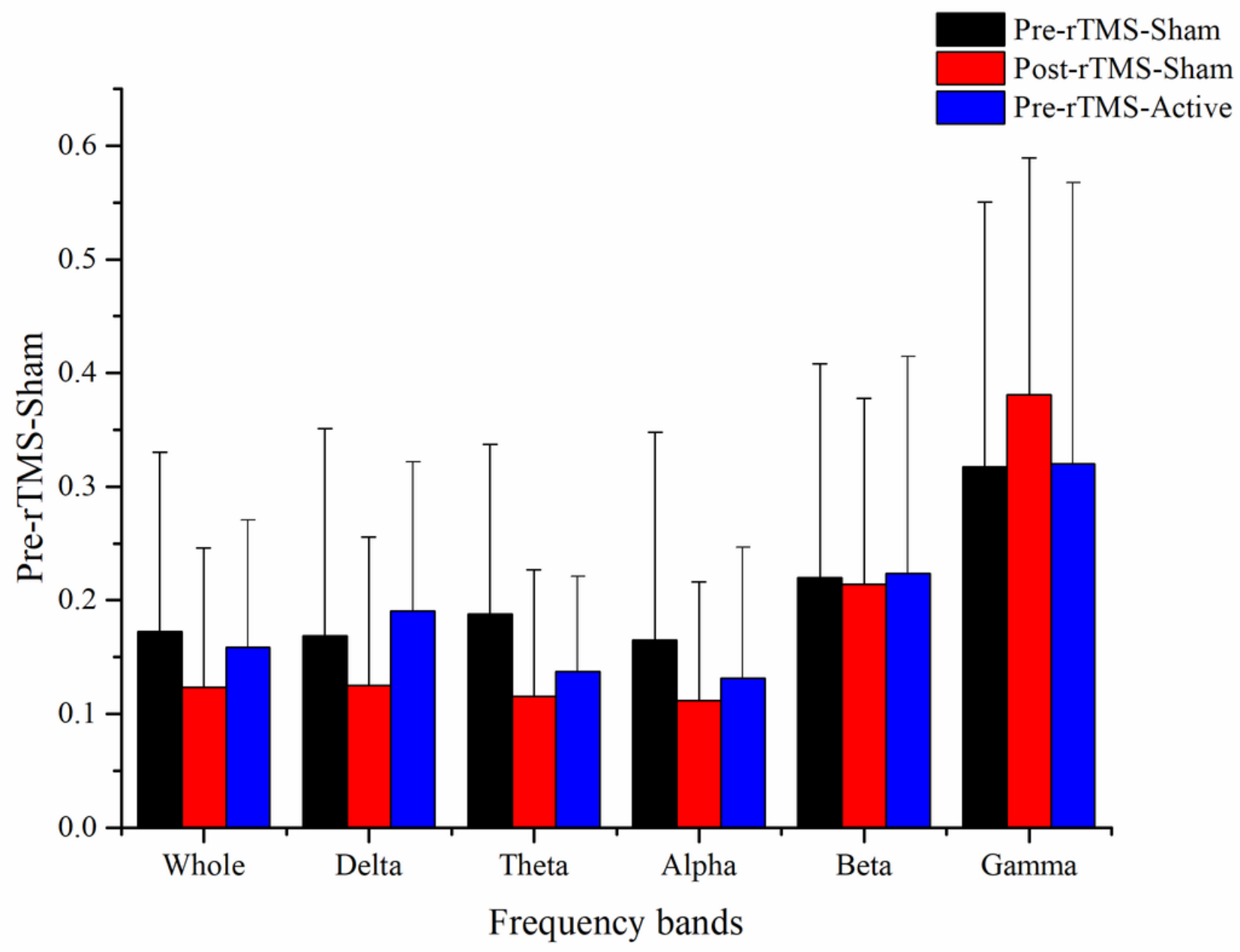

Figure 1

The comparison between sham and active sessions. No significant difference in BSI was found among the prerTMS and post-rTMS in the sham-stimulation sessions and the pre-rTMS in the active sessions. 

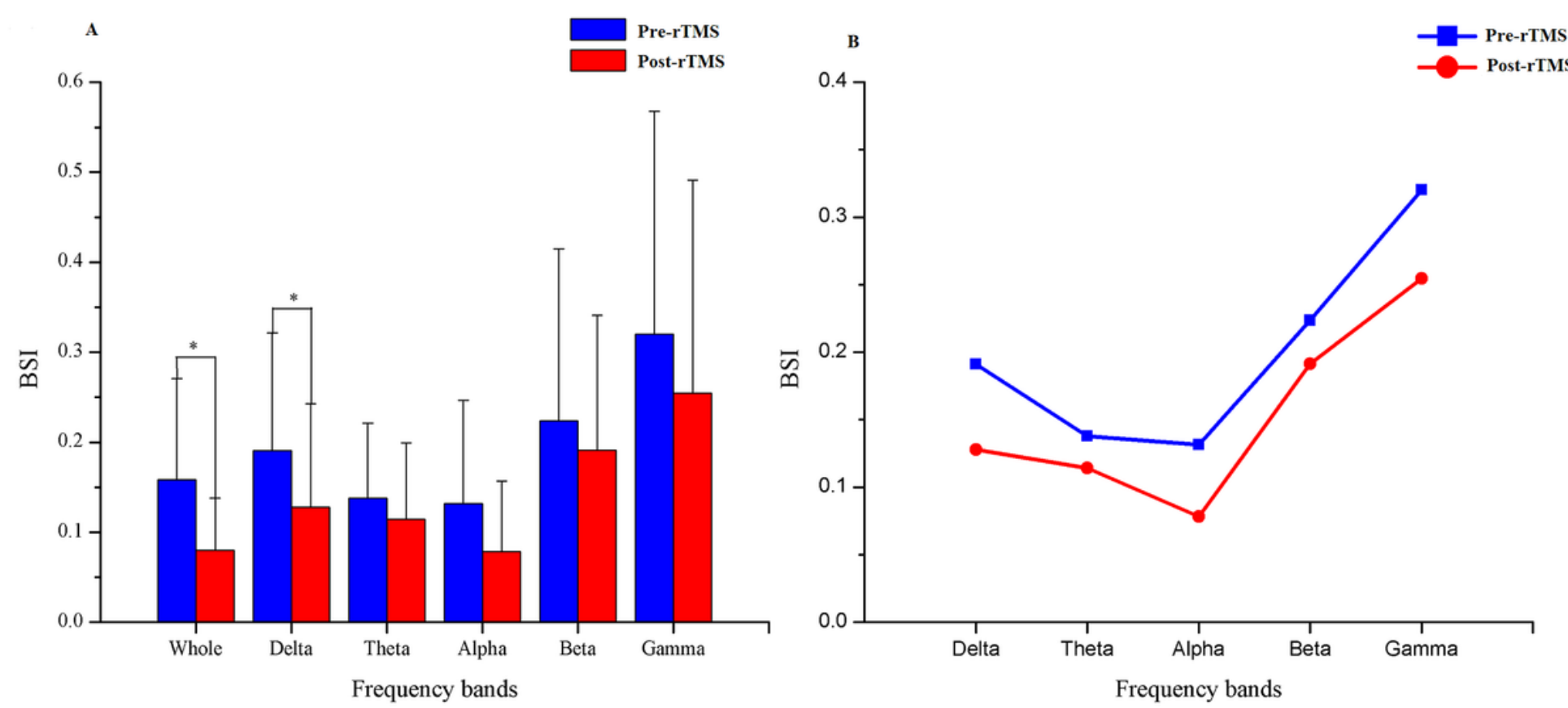

Figure 2

Interhemispheric BSI. The interhemispheric asymmetry of the whole frequency range in pre-rTMS was significantly less than in post-rTMS (A). At different frequency bands, the BSI was significantly decreased only in the delta band. The curve on the graph shows the BSI difference among the five frequency bands (B). An asterisk refers to a significant difference in the pre-post comparison with a P-value below 0.05.
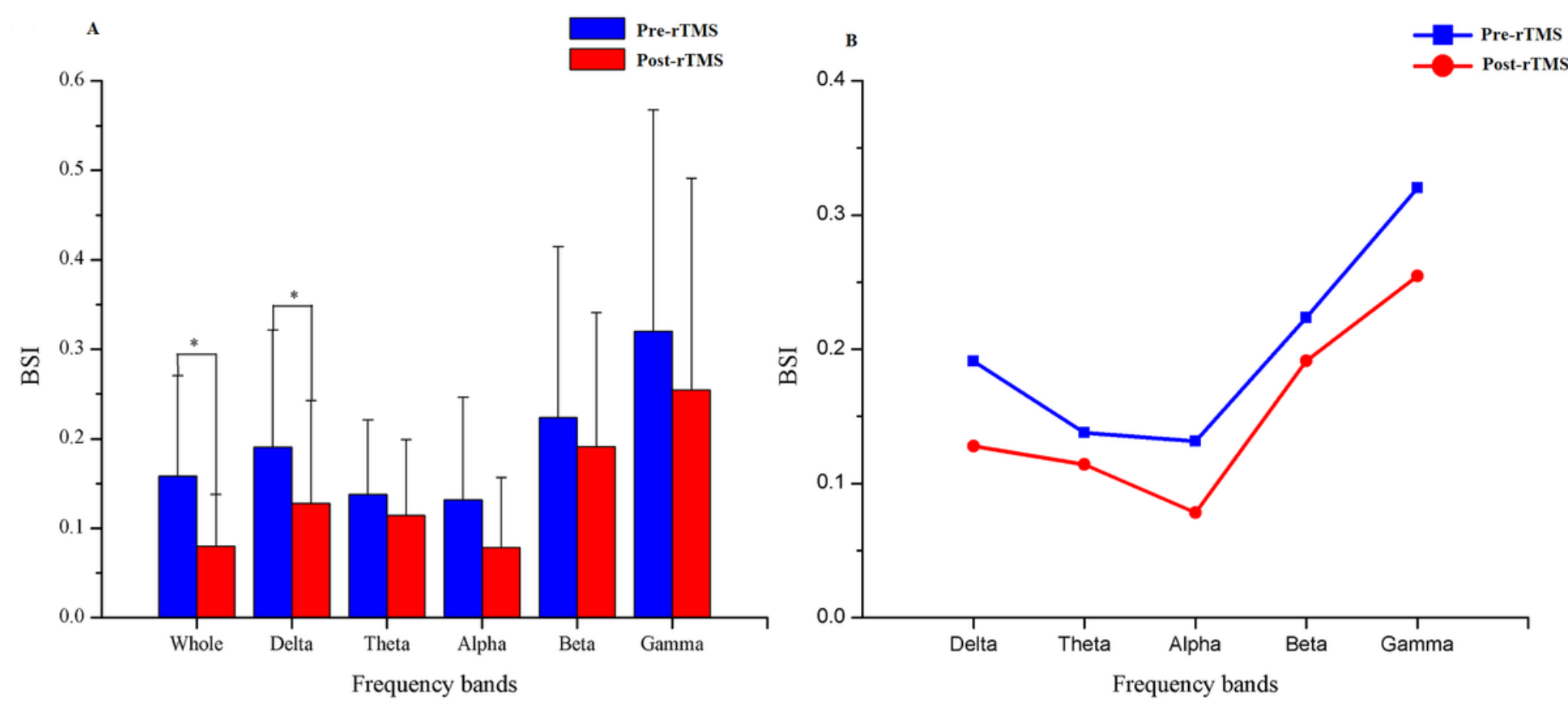

Figure 2

Interhemispheric BSI. The interhemispheric asymmetry of the whole frequency range in pre-rTMS was significantly less than in post-rTMS (A). At different frequency bands, the BSI was significantly decreased only 
in the delta band. The curve on the graph shows the BSI difference among the five frequency bands (B). An asterisk refers to a significant difference in the pre-post comparison with a P-value below 0.05.
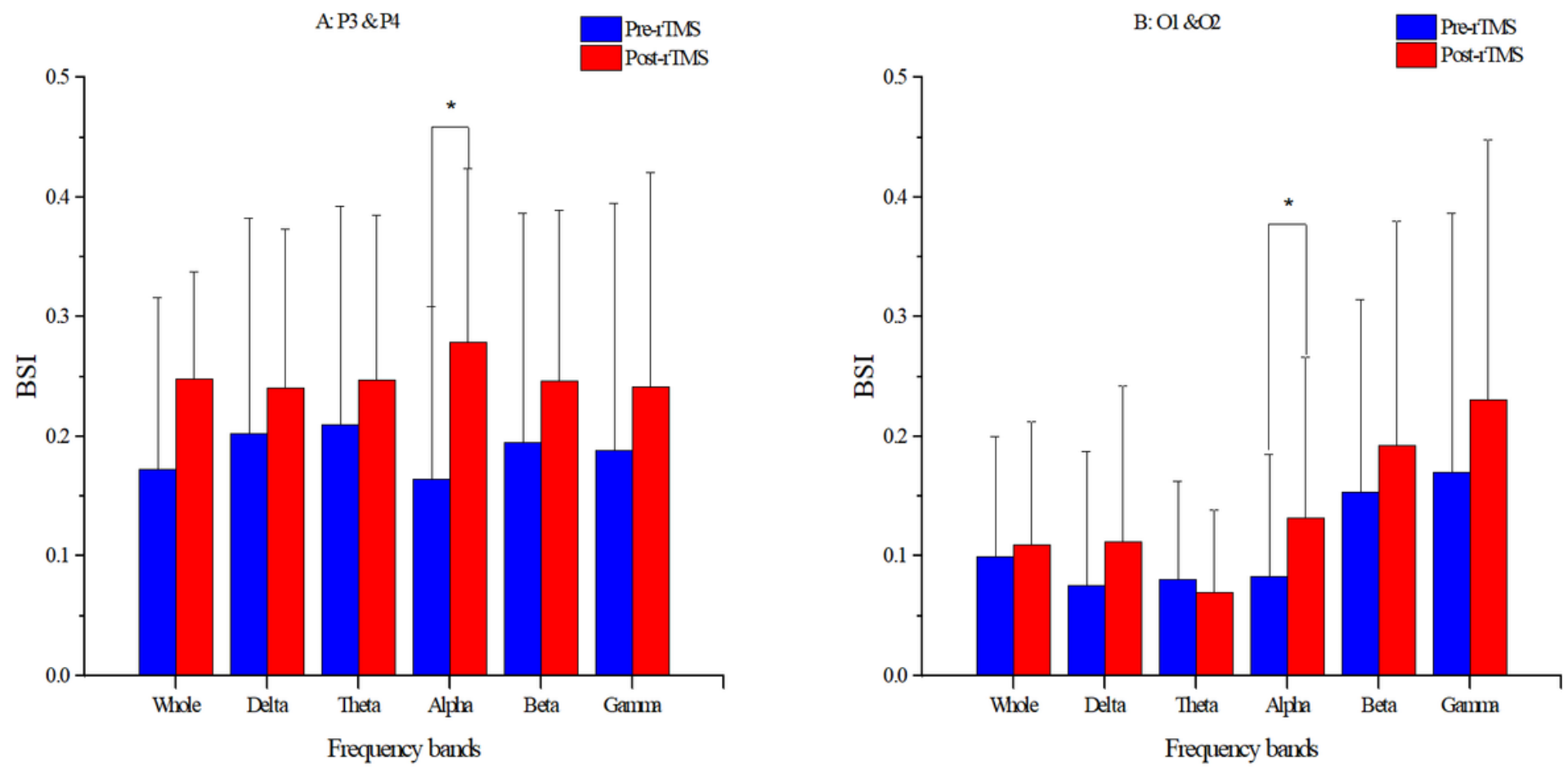

Figure 3

The BSI in paired channels. We only show channels P3 \& P4 and 01 \& 02 here. A significant difference in the alpha bands was found in channels P3 \& P4 and 01 \& 02. The BSI in pre-rTMS was significantly lower than in post-rTMS. An asterisk denotes a significant difference with a P-value below 0.05. Other channels can be found in the supplementary files (https://figshare.com/articles/BSI_in_paired_channels_xlsx/11956332).
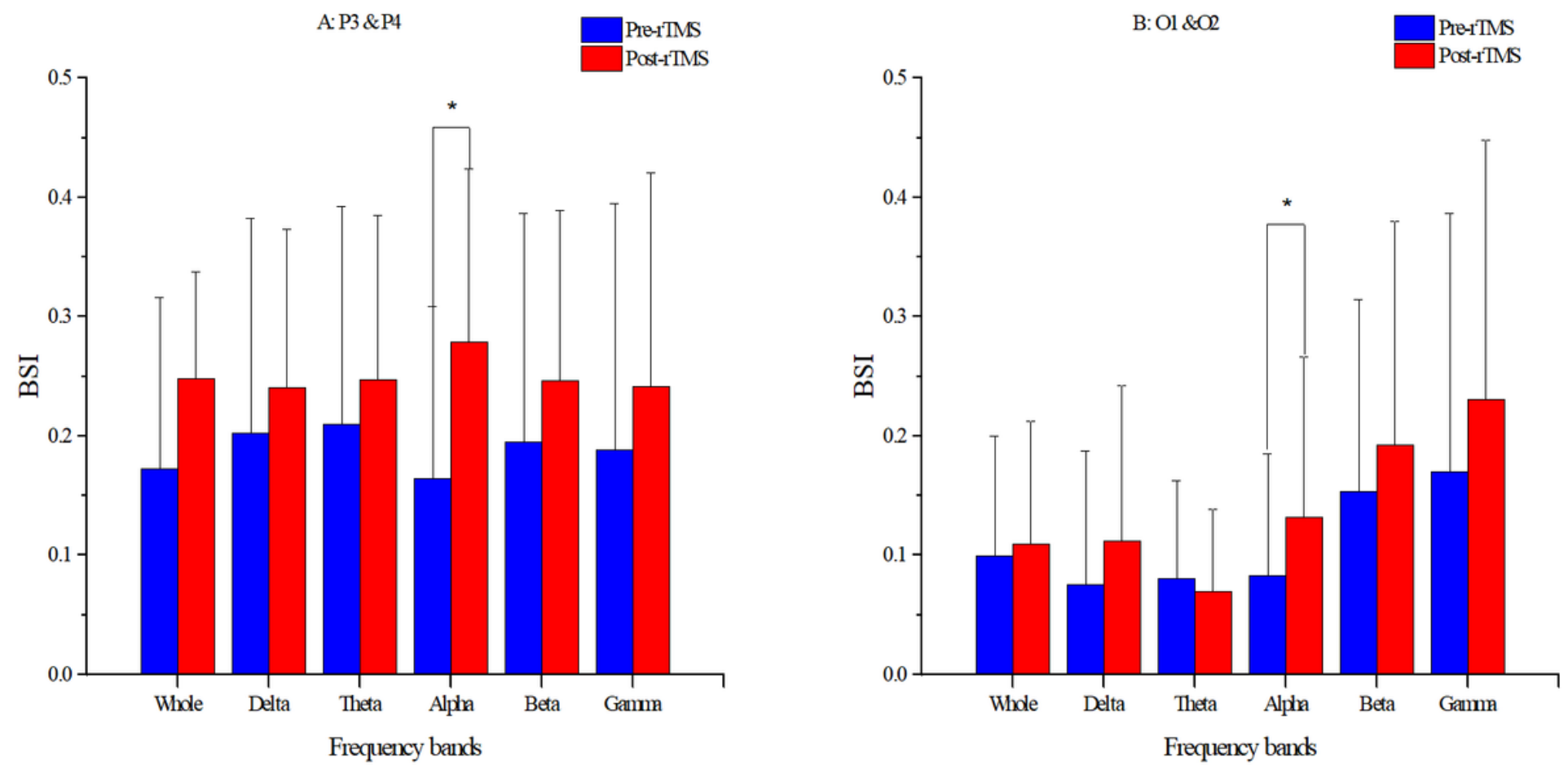


\section{Figure 3}

The BSI in paired channels. We only show channels P3 \& P4 and $01 \& 02$ here. A significant difference in the alpha bands was found in channels P3 \& P4 and 01 \& 02 . The BSI in pre-rTMS was significantly lower than in post-rTMS. An asterisk denotes a significant difference with a P-value below 0.05 . Other channels can be found in the supplementary files (https://figshare.com/articles/BSI_in_paired_channels_xlsx/11956332).
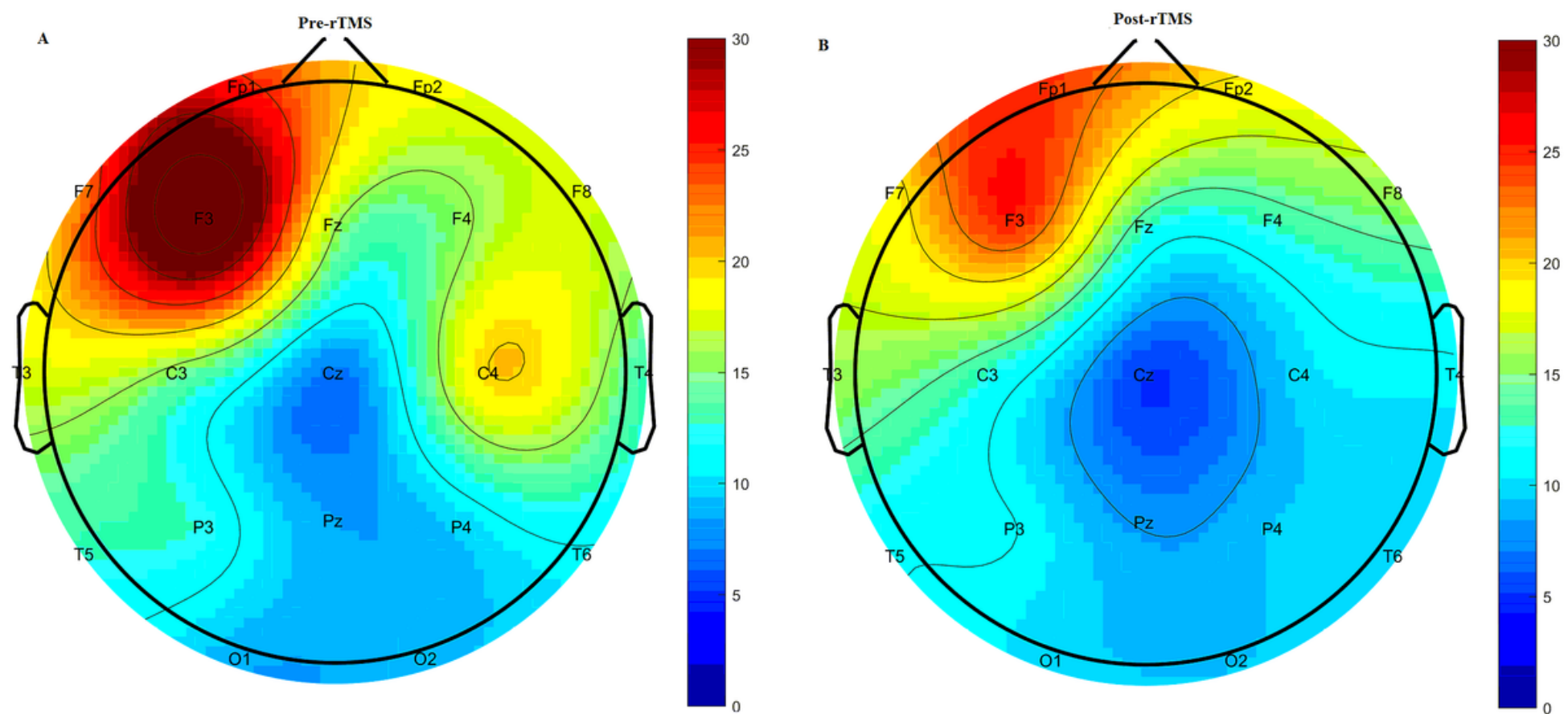

c

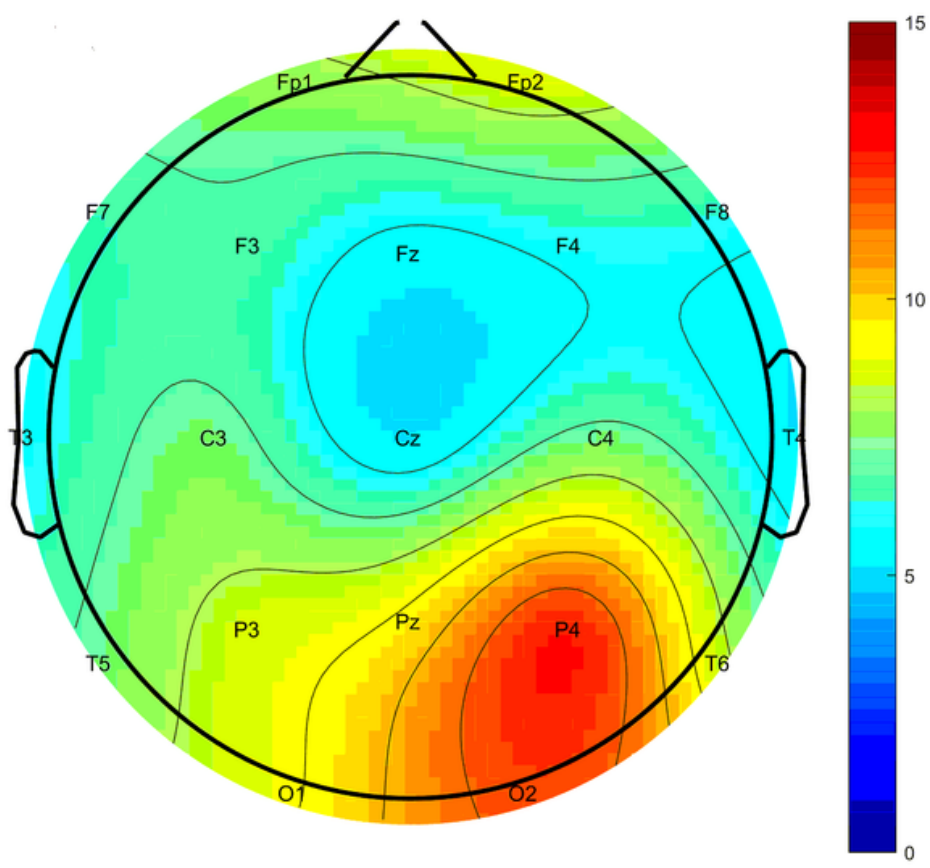

D

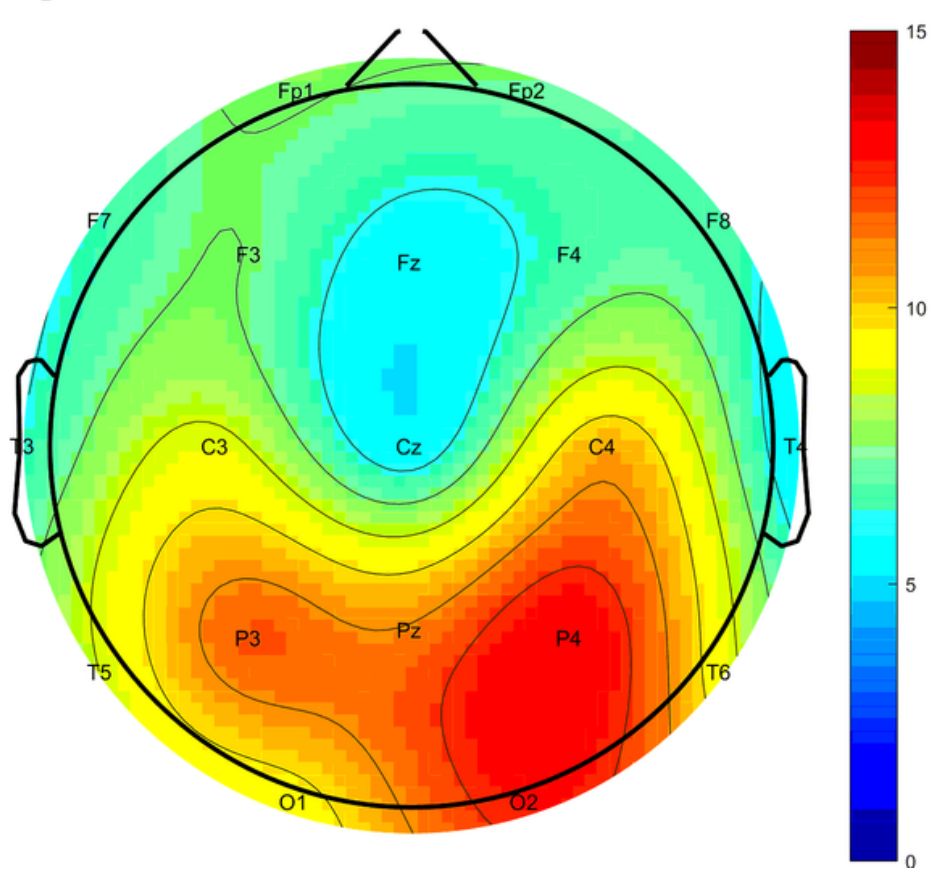

Figure 4

Topographical distribution of pre-post rTMS. The left hemisphere is the lesioned side. The left column is prerTMS (A \& C) and the right column is post-rTMS (B \& D). The topographical distribution of delta power focused 
on the left side and showed a visible reduction after rTMS (A). Oscillations in the alpha band were more prominent and mainly distributed in the parietal-occipital area (B).
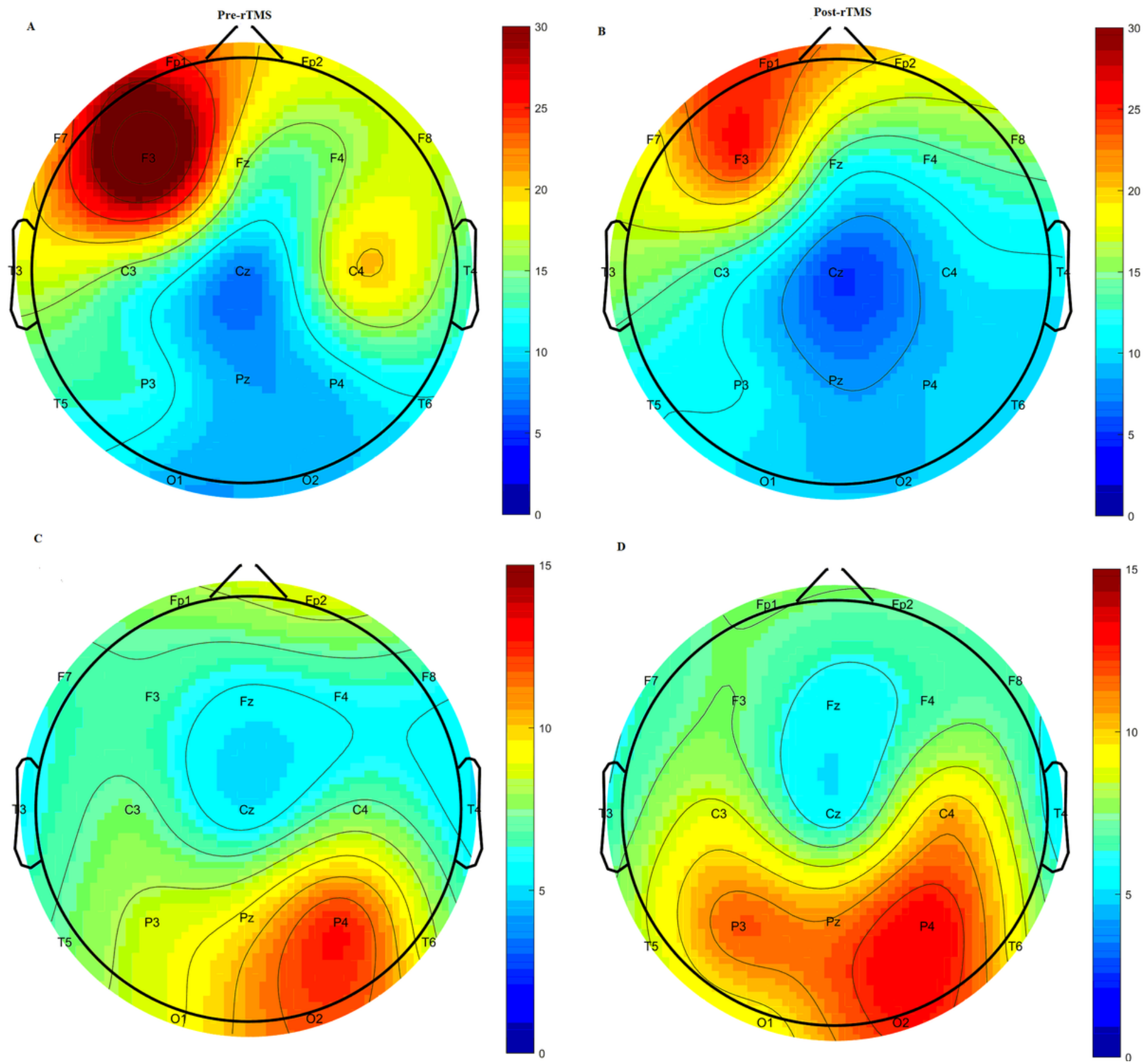

Figure 4

Topographical distribution of pre-post rTMS. The left hemisphere is the lesioned side. The left column is prerTMS (A \& C) and the right column is post-rTMS (B \& D). The topographical distribution of delta power focused on the left side and showed a visible reduction after rTMS (A). Oscillations in the alpha band were more prominent and mainly distributed in the parietal-occipital area (B). 